\title{
Paleocontraintes et Contexte Tectonique de Mise en Place du Volcanisme Alcalin Neogene et Quaternaire du Moyen Atlas (Maroc)
}

\author{
Saïd Hinaje, \\ Mohamed El Fartati, \\ Driss Yaagoub, \\ Samir Amrani, \\ Youssef Gharmane,
}

Laboratoire de Géo-ressources et Environnement, Université Sidi Mohamed

Ben Abdellah, Faculté des Sciences et Techniques, Fès, Maroc

Bouhsine El Fellah Idrissi,

Laboratoire des Sciences de la Vie et de la Terre, Centre Régional des

Métiers de l'Éducation et de la Formation, El Jadida, Maroc

Doi: 10.19044/esj.2019.v15n15p448 URL:http://dx.doi.org/10.19044/esj.2019.v15n15p448

\section{Résumé}

Au cours de la période néogène et quaternaire, le Moyen Atlas a connu une activité volcanique en relation avec des phénomènes magmatiques et tectoniques complexes. Plusieurs dizaines d'édifices volcaniques appartiennent aux types stromboliens et maars. Ce volcanisme a commencé au Miocène par des coulées, des sills, des dykes et des culots de nature alcaline. Les produits pyroclastiques émis correspondent à des basaltes alcalins, à des basanites et à des néphélinites. En raison des relations morphologiques entre le réseau hydrographique actuel, les dernières laves volcaniques et les grabens subméridiens; les basaltes du Moyen Atlas ont été attribués, sans argument structural, à la dernière phase tectonique du Quaternaire. Le but de cette note est d'exposer les résultats de l'étude de la répartition des volcans suivant les alignements tectoniques en relation avec les phases de déformation. Les édifices volcaniques sont alignés selon les directions N130, N70, N40 et N170, avec une prédominance de la direction N130. Un exemple de ce dernier alignement est la zone volcanique Timahdite-Azrou que nous interprétons comme un graben orienté NW-SE, à remplissage fluvio-lacustre au Miocène supérieur. Un autre exemple d'activité volcanique est celle liée à des ouvertures en transtension dans les zones de jonction entre des failles antithétiques (nœuds tectoniques); comme les volcans de Zelghi, d'Aguelmam Sidi Ali, de Oum Larays et de Tafraoute-Aït Bouziane. Les volcans les plus 
récents sont associés aux failles normales subméridiennes d'âge quaternaire moyen-récent, comme ceux d'Azghar-Boutferoun situés dans les causses d'Anouceur et d'Amekla.

Mots-clés : Volcanisme, tectonique cassante, paléo-contraintes, Néogène, Quaternaire, Moyen Atlas, Maroc

\title{
Palaeostresses and Tectonic Context of Setting Up Neogene Quaternary Alkali Volcanism of the Middle Atlas (Morocco)
}

\author{
Saïd Hinaje, \\ Mohamed El Fartati, \\ Driss Yaagoub, \\ Samir Amrani, \\ Youssef Gharmane,
}

Laboratoire de Géo-ressources et Environnement, Université Sidi Mohamed

Ben Abdellah, Faculté des Sciences et Techniques, Fès, Maroc

\section{Bouhsine El Fellah Idrissi,}

Laboratoire des Sciences de la Vie et de la Terre, Centre Régional des

Métiers de l'Éducation et de la Formation, El Jadida, Maroc

\section{Abstract}

During the Neogene and Quaternary periods, the Middle Atlas was characterized by a volcanic activity in relation to complex magmatic-tectonic phenomena. Volcanoes belong to the strombolian and maar types. This volcanic activity began in the Miocene by alkaline flows, sills, dykes and volcanic caps. The pyroclastic rocks emitted by volcanoes correspond to alkali basalts, basanites and nephelinites. Because of the morphological relationships existing between the recent hydrographic system, the last volcanic lavas and the sub-meridian grabens; the Middle Atlas basalts have been assigned, without field structural arguments, to the last Quaternary tectonic phase. The purpose of this paper is to expose the results of the study of the distribution of volcanoes in relation to the tectonic alignments. The volcanic edifices are aligned along the N130, N70, N40 and N170 directions, with a predominance of the N130 trend. For example, the Timahdite-Azrou volcanic zone can be interpreted as a NW-SE graben filled with Late Miocene fluvial and lacustrine 
deposits. Another example of volcanic activity related to transtensional openings at the junction zones between antithetic faults (tectonic nodes) can be found around the Zelghi, Aguelmam Sidi Ali, Oum Larays and TafraouteAit Bouziane volcanoes. The most recent volcanoes are associated with middle - late Quaternary normal sub-meridian faults like the volcanoes of Azghar-Boutferoun located in the Anoceur and Amekla plateau (causses).

Keywords: Volcanism, brittle tectonic, palaeostresses, Neogene, Quaternary, Middle Atlas, Morocco

\section{Introduction}

Au cours de la période néogène et quaternaire, le Moyen Atlas, comme le reste du domaine atlaso-mesetien et le domaine rifain, a connu une intense activité volcanique. A l'échelle du Maroc, cette activité caractérise tout le territoire depuis le Siroua au SW au Gourougou au NE le long de la « Moroccan Hot Line » (Frizon de Lamotte et al., 2009). Dans le Moyen Atlas, les appareils volcaniques de type strombolien ou maar, sont liés à des failles à jeux polyphasés (Martin, 1981) (Figure 1). Les différentes activités volcaniques sont liées à des épisodes tectoniques qui s'échelonnent depuis le Miocène (Vallésien) jusqu'au Quaternaire moyen-récent (Charrière, 1990; Hinaje et Aït Brahim, 2001; Hinaje, 2004). Ces activités sont caractérisées par des coulées, des sills, des dykes et des culots de nature alcalines (Termier et al., 1948 ; Choubert et al., 1965 ; Jaeger et al., 1973 ; Harmand et Cantagrel, 1984 ; Harmand et Moukadiri, 1986 ; Chorowicz et al., 2001 ; El Azzouzi et al., 2010).

Dans la bordure septentrionale du bassin de Guercif (bord NE du Moyen Atlas), le volcanisme calco-alcalin à shoshonitique est lié à une tectonique distensive lors de l'ouverture du bassin au cours du Miocène supérieur (Hernandez et Bellon, 1985 ; Charroud, 2002). Ce volcanisme est suivi par une autre activité volcanique alcaline d'âge plio-quaternaire (Torbi, 1997).

Dans le Rif (région de Nador), il y a trois périodes d'activités volcaniques (Kerchaoui, 1994) :

- la première d'âge miocène moyen (14 - 10 M. a.), a conduit à la mise en place des andésites de Ras Tarf;

- la seconde d'âge miocène supérieur (10 - 4 M. a.) est représentée par une série calco-alcaline ou shoshonitique ;

- la troisième d'âge plio-quaternaire (3 - 2 M. a.) est de nature alcaline.

Ces activités volcaniques sont marquées par des dykes, des sills, des necks et des tufs dont les directions des alignements sont variables (N-S, NESW et NW-SE) (Chotin et Aït Brahim, 1988). Dans cette zone rifaine, 
l'activité volcanique d'âge miocène est liée aux décrochements senestres N40 et N70. Par contre du Messinien à l'Actuel, elle est liée selon ces auteurs, à des décrochements dextres N90 et à des décrochements senestres subméridiens. Les périodes d'arrêt de l'activité volcanique pourraient correspondre aux périodes de rotation des directions des contraintes tectoniques (Chotin et Ait Brahim, 1988).

Dans le Moyen Atlas et la Haute Moulouya, l'activité volcanique s'est manifestée en trois périodes majeures (Harmand et Cantagrel, 1984). La plus ancienne d'âge éocène, se situe sur la bordure NE du Haut Atlas (massif à carbonatites de Bou Agrao). Une deuxième période d'activité volcanique est représentée par un volcanisme diffus d'âge miocène moyen - supérieur (15-6 M.a.) ou 16,25-5,87 M.a. pour M. El Azzouzi et al. (2010). La dernière période d'âge quaternaire moyen (1,8-0,5 M.a.), à plio-quaternaire (3,77-0,60 M.a)(El Azzouzi et al., 2010), est-elle même divisée en plusieurs épisodes volcaniques. Par exemple le maar Lechmine N'Kettane, où les dépôts volcano-détritiques rejetés vers la périphérie, sont recouverts par les coulées basaltiques issues du volcan de Jbel Habri daté par les mêmes auteurs à $0,8 \pm 0,25$ M.a., et à $0,76 \pm$ 0,07 M.a. Nous avons constaté que les cendres volcaniques issues de ce dernier volcan, sont recouvertes à leur tour par des dépôts volcano-sédimentaires d'un petit maar situé entre Jbel Habri et Tichout Tazouggaght (Hinaje, 2004). Ces dernières activités correspondent à un volcanisme alcalin qui résulterait de déformations du manteau supérieur responsables d'une fusion partielle (Harmand et Moukadiri, 1986). Les appareils volcaniques sont en majorité des cônes de scories, précédés parfois par des maars. Les édifices volcaniques sont localisés sur des fractures N40 à N50, selon deux alignements N70 et N170 (Harmand et Moukadiri, 1986). Les produits volcaniques provenant des cônes et des maars, sont caractérisés par la présence d'enclaves ultrabasiques et basiques (Moukadiri, 1983).

\section{Contexte géologique}

Depuis le Miocène moyen jusqu'au Quaternaire, le Maghreb est affecté par plusieurs périodes d'activités volcaniques caractérisées par une évolution des séries magmatiques de nature calco-alcaline au Miocène moyen, calco-alcaline et alcaline au Miocène supérieur et alcaline à la fin du Messinien et au Plio-Quaternaire (Piqué et al., 1998). Cette évolution montre que les sources du volcanisme sont mantelliques asthénosphériques, avec deux types de réservoirs ; l'un dérivé du manteau et l'autre crustal (El Azzouzi et al., 1999 ; 2010 ; Duggen et al., 2009). Dans le pourtour de la Méditerranée, ces derniers auteurs différencient quatre périodes d'activité volcaniques. La première d'âge Langhien (16 à $15 \mathrm{M}$. a.) et la deuxième d'âge Serravalien (14,5 à 11 M. a.), sont de nature calco-alcaline (Bellon, 1981). La troisième (9 à 4 M.a.: Tortonien-Pliocène inférieur) est de nature shoshonitique (plus riche 
en calcium). La dernière est de nature alcaline évoluant du Messinien au Quaternaire. D’après nos résultats (Hinaje, 2004 ; ce travail), cette dernière activité volcanique alcaline est synchrone avec plusieurs phases tectoniques divisées en épisodes. Une extension NE-SW au Tortonien supérieurMessinien, une compression NW-SE au Pliocène inférieur-moyen, une extension NNW-SSE au Pliocène moyen-supérieur, une compression N30 associée à une extension N120 au Quaternaire ancien-moyen et une compression N170 associée à une extension N80 au Quaternaire moyenrécent. Il faut noter qu'au cours de chaque phase, des permutations entre les axes de contraintes $\sigma 1-\sigma 2$ et $\sigma 2-\sigma 3$, dues aux variations de leurs intensités, définissent les épisodes tectoniques qui constituent une phase de déformation cassante.

\section{Matériels et méthodes}

Le Moyen Atlas est le siège d'une activité tectonique, néotectonique et sismique importante (Michard, 1976 ; Ramdani et Tadili, 1980 ; Charrière, 1990; Hinaje, 2004), que nous avons étudié par le biais des différents marqueurs microtectoniques. Des centaines de mesures microtectoniques ont été recueillies dans les formations néogènes et quaternaires du Moyen Atlas (Hinaje et al., 2015). Ces mesures ont été traitées ensuite par un ensemble de programmes informatiques (Angelier et Mechler, 1977 ; Angelier, 1990, 1991).

Sur les sites de mesures, les critères retenus pour caractériser la déformation cassante pendant une période précise sont les suivants :

- l'âge des terrains fracturés,

- les décalages des fractures par d'autres,

- la superposition des stries sur les miroirs des failles,

- la présence de fractures parallèles à jeux incompatibles,

- les failles synsédimentaires ou synvolcaniques scellées, dont les miroirs ne montrent pas de recristallisations,

- principe d'élimination; de l'épisode tectonique le plus récent vers le plus ancien.

Les mesures de terrains comportent : directions et pendages des failles, pitch des stries (contrainte cisaillante ou vecteur glissement) et sens des jeux. Les programmes informatiques de calcul du tenseur de contrainte d'Angelier (version 1990-91) proposent cinq méthodes de calcul :

- les méthodes (R2DT) et (R2DS) assurent une analyse

bidimensionnelle,

- les méthodes (R4DT) et (R4DS) permettent une analyse quadridimensionnelle.

Ces quatre méthodes sont basées sur le calcul et la comparaison des différents tenseurs, puis ne retiennent que ceux qui minimisent une fonction simple de l'angle entre la contrainte cisaillante calculée et le vecteur glissement observé. Ainsi le nombre de mesure se trouve réduit à celles dont 
l'écart angulaire entre la strie mesurée et celle calculée est inférieur à $20^{\circ}$. Cette opération est répétée pour chaque mesure, ce qui rend le traitement long (Angelier, $1975 ; 1984$ ).

La cinquième méthode dite inversion directe (INVDIR) est basée sur :

- l'angle d'écart entre strie réelle et strie calculée inférieur à 22,5 (ANG $<22,5$ )

- la magnitude relative de la contrainte cisaillante (RUP) qui doit être inférieure à 50\% (RUP < 50\%), pour vaincre la friction et la cohésion sur les plans de failles.

Les cinq méthodes de traitement donnent les mêmes résultats, sauf que la méthode «INVDIR » a l'avantage d'un calcul rapide. C'est pour cette raison que nous l'avons utilisée dans nos travaux.

\section{La province volcanique d'Azrou-Timahdite}

Cette zone regroupe l'essentiel des édifices volcaniques de type maar et de type strombolien (conique) (Figure 1). Parfois ces deux types d'appareils se trouvent superposés, comme le cas du volcan de Timahdite. Sur le terrain on distingue trois types de structures volcaniques, parfois superposés, et dont les activités sont successives :

- les cratères en dépression ou maar et leurs dépôts volcano-détritiques situés à leurs périphéries ;

- les cônes de scories contenant des dépôts pyroclastiques de différents types ;

- les coulées fluides qui ont emprunté les vallées et les dépressions sur plusieurs kilomètres. C'est le cas des coulées du volcan Outgui qui arrivent jusqu'à la plaine de Saïs, et celles des volcans d'Aguelmam Sidi Ali et de Timahdit qui ont suivi la vallée de Oued Guigou. 

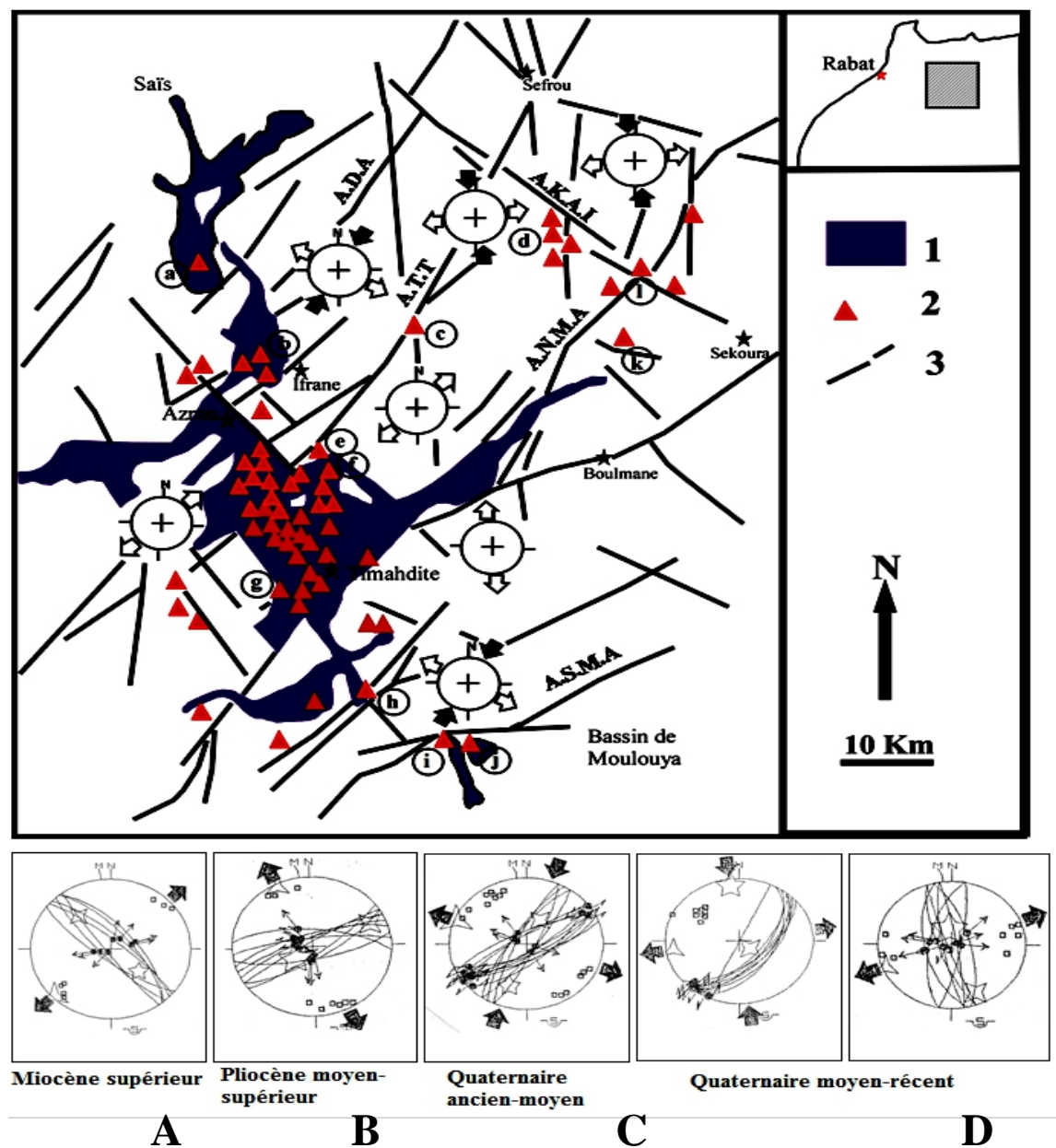

Figure 1 : carte des principales failles et des édifices volcaniques néogènes et quaternaires du Moyen Atlas, et paléocontraintes génératrices de ce volcanisme

1- laves basaltiques ; 2- cratères volcaniques (maars et cônes) ; 3principales failles. A.D.A : accident de Dayet Aoua ; A.T.T : accident de Tizi N'Tretten ; A.N.M.A : accident nord moyen atlasique ; A.S.M.A : accident sud moyen atlasique ; A.K.A.I : accident de Kandar-Amane Ilila ; a- volcan Outgui ; b- El Koudiat ; c- Reggada ; d- Azghar ; e- Michlifène ; f- Lechmine N'Kettane ; g- Timahdit ; h- Aguelmam Sidi Ali ; i: Oum Larays ; jLougunina ; k- Tafraout ; 1- Zelghi. A, B, C et D : stéréogrammes contenant les traces cyclographiques, les traces polaires des failles et les états de contraintes responsables de la genèse et de la géométrie des alignements volcaniques néogènes et quaternaires du Moyen Atlas. (Flèche centrifuge : extension; flèche centripète : compression; étoiles à 3 , 4 et 5 branches représentent respectivement les contraintes $\sigma 3, \sigma 2$ et $\sigma 1 ; \mathrm{M} \mathrm{N}$ : Nord 
magnétique et Nord géographique (diagramme de schmidt, hémisphère inférieur)).

La plupart des produits volcaniques sont masqués par des épanchements plus récents. Chaque série de volcans possède une activité volcanique qui peut être de plusieurs milliers voir des millions d'années, marquée par des périodes d'arrêt et des périodes de reprise. Généralement, les datations réalisées par nos prédécesseurs, ont été faites sur la dernière activité volcanique représentée par des laves basaltiques fluides (Harmand et Cantagrel, 1984; El Azzouzi et al., 1999-2010). Les âges obtenus correspondent aux derniers épisodes volcaniques qui précèdent l'arrêt de cette activité. Par contre aucune datation n'a été faite sur des produits volcaniques provenant des volcans de type maar. Ceux-ci sont souvent antérieurs aux cratères de forme conique. C'est le cas des maars de Timahdite et de Lechmine N'Kettane dont les produits volcaniques sont recouverts par des laves et des dépôts pyroclastiques provenant des cratères coniques avoisinants et/ou en superposition.

Dans le volcan de type maar de Lechmine N'Kettane, situé dans la province volcanique d'Azrou - Timahdite (Figure 1), l'analyse lithostratigraphique des dépôts volcano-détritiques rejetés par ce maar montre une succession de niveaux détritiques fins et grossiers. Ceux-ci sont constitués par un conglomérat bréchique polygénique et hétérométrique, contenant des blocs liasiques arrondis. Les éléments sont constitués par du calcaire grisâtre, du calcaire cuit blanchâtre et du basalte. La matrice est sableuse à microconglomératique, de même nature lithologique que les éléments. L'ensemble est agencé en plusieurs séquences rythmiques de 10 à $30 \mathrm{~cm}$ d'épaisseur, constituées par deux niveaux : un niveau grossier à la base et un niveau fin au sommet (Figure 2). Chaque séquence correspond à une explosion qui rejette à la fois des éléments anguleux provenant du substratum, des éléments arrondis et du basalte. Les éléments liasiques de forme sub-arrondie à arrondie, dont la taille dépasse parfois $1 \mathrm{~m}$ (Figure 3), témoignent de l'effondrement de la zone et de son remplissage par des dépôts fluviatiles et probablement lacustres, avant les explosions volcaniques. La taille grossière des éléments arrondis témoigne du long transport des dépôts fluviatiles déposés dans ce bassin subsident orienté NW-SE. Les dépôts volcano-détritiques de type maar, possédant un pendage vers l'extérieur du cratère, sont recouverts par des coulées basaltiques provenant des volcans avoisinants de forme conique. Ces dépôts volcano-détritiques sont postérieurs au jeu normal d'une faille N140 qui limite ce volcan du côté NE. Le prolongement de cette faille, limite tout le bassin effondré d'Azrou-Timahdite de direction NW-SE, probablement à remplissage sédimentaire fluvio-lacustre, qui est antérieur aux activités volcaniques. En témoigne la présence de blocs liasiques métriques arrondis d'origine torrentielle, dans les dépôts rejetés par le maar. De point de vue 
tectonique, ces dépôts sont affectés par des failles normales subméridiennes dont les rejets sont faibles et les miroirs sont tapissés par une croûte calcaire (Figure 3). Ces failles témoignent d'un régime tectonique extensif ENE-WSW d'âge quaternaire moyen-récent (Hinaje, 2004).

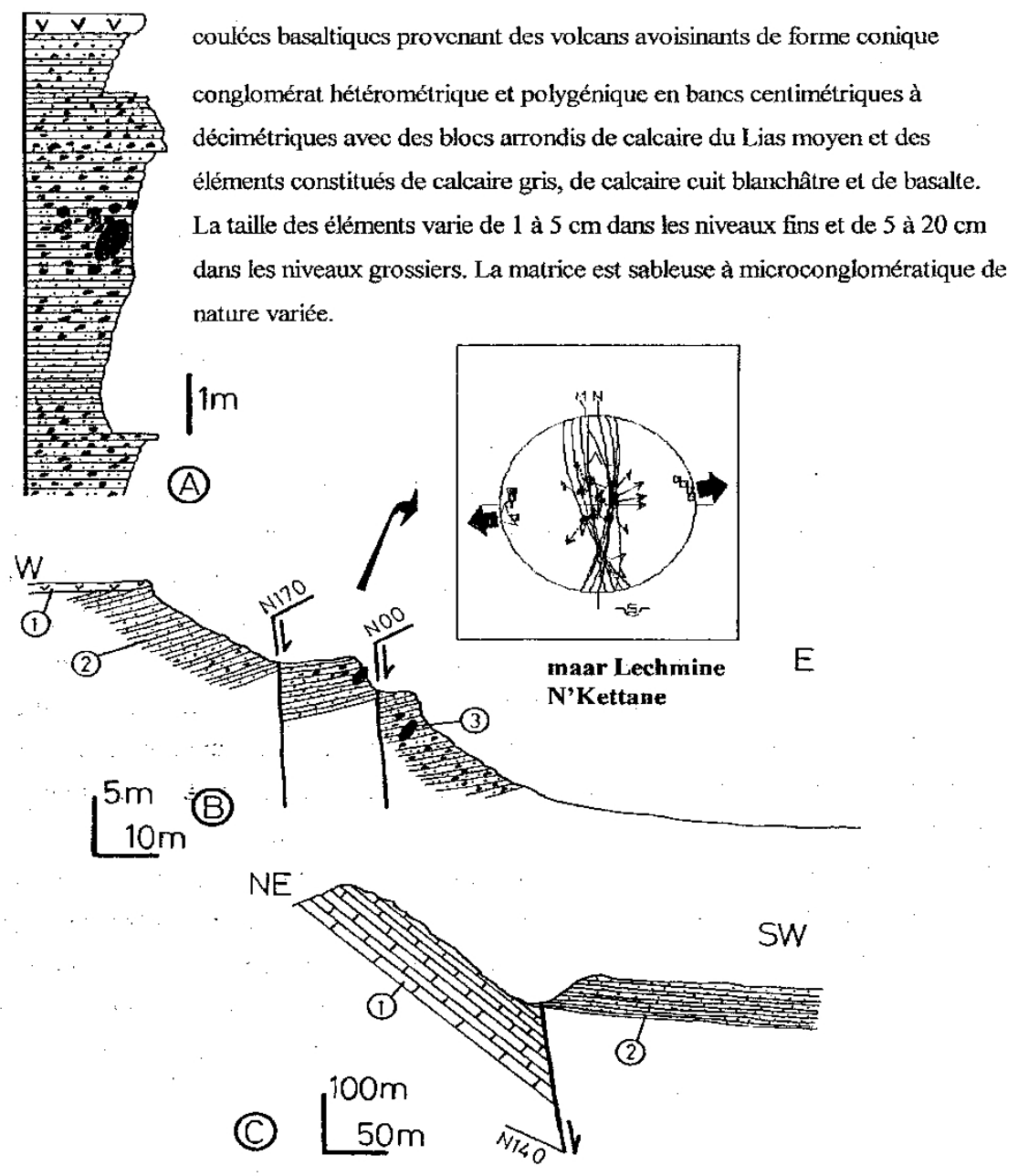

Figure 2: A- Coupe lithostratigraphique des dépôts volcano-détritiques du maar Lechmine N'Kettane. B- Coupe structurale au niveau du bord occidental du maar. 1- basaltes en coulées ; 2- dépôts volcano-détritiques de type maar ; 3- élément liasique arrondi de taille métrique. C- Coupe structurale montrant l'effondrement des couches du Lias moyen par une faille normale N140. 1- calcaire lité du Lias moyen ; 2- dépôts volcano-détritiques de type maar, postérieurs au jeu normal de la faille N140. 

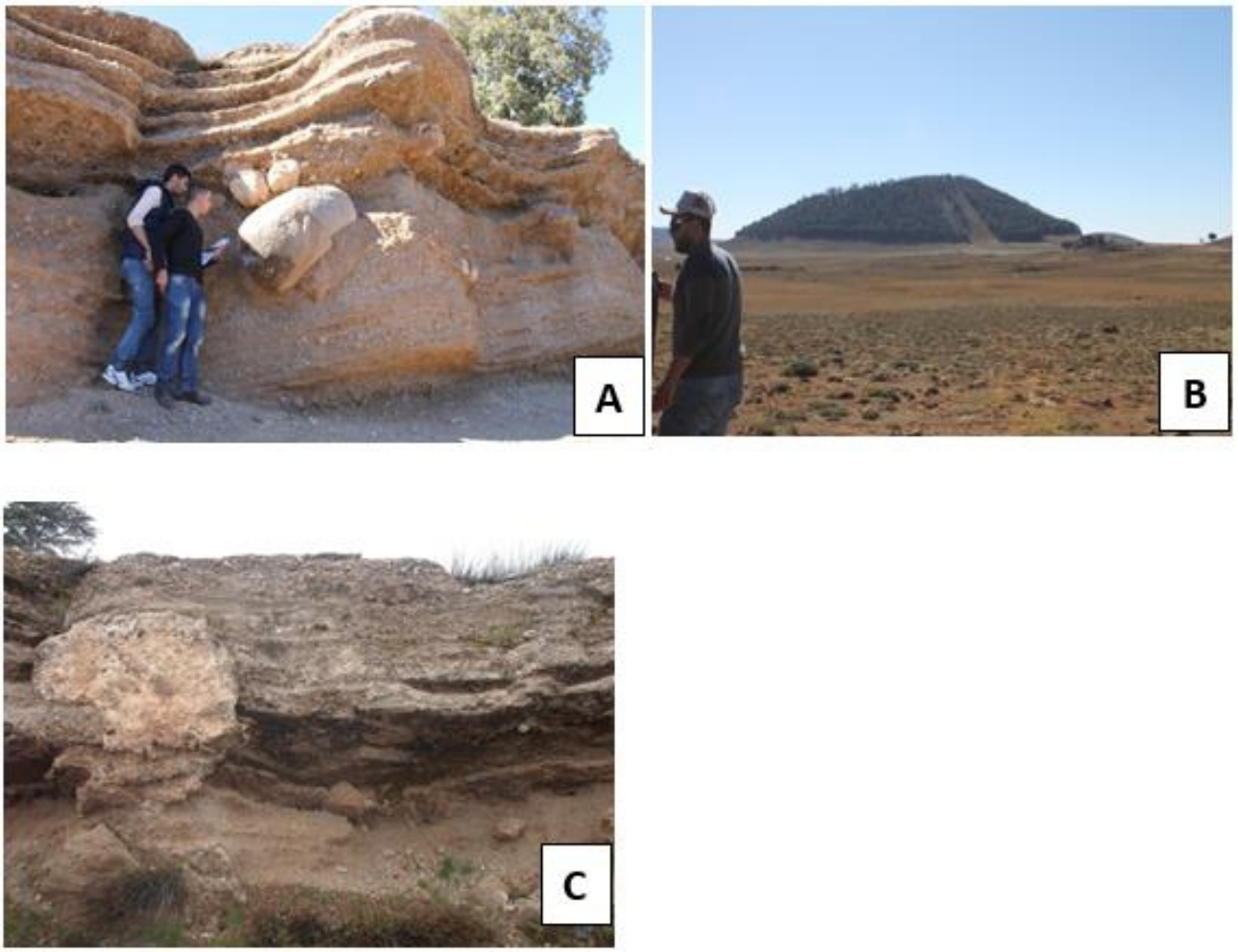

Figure 3 : A- dépôts volcano-sédimentaires du maar Lechmine N'Kettane, contenant des éléments carbonatés liasiques arrondis, de taille décimétrique à métrique. B- Cratère volcanique de Habri. C- Plan de faille N170 encroûté, affectant les dépôts volcanosédimentaires du maar Lechmine N'Kettane.

Le volcan de Timahdite possède la particularité de la superposition dans l'espace et dans le temps de deux activités volcaniques. La première est de type maar; marquée par des dépôts conglomératiques contenant des éléments constitués de calcaire rose de l'Eocène, et de basalte. La seconde correspond à un cône volcanique constitué de scories, de tufs, de bombes et de laves cordées. L'âge de cette dernière activité volcanique est de $0,67 \pm 0,09$ M.a. (El Azzouzi et al., 2010). Le temps qui sépare les deux activités n'est pas connu, car le volcan sous-jacent de type maar n'est pas daté. Les dépôts volcano-détritiques de ce maar sont affectés par quelques micro-failles normales synvolcaniques (scellées), orientées NW-SE. Le traitement stéréographique de ces failles donne un état de contraintes extensif, tels que l'axe $\sigma 1$ est vertical et l'axe $\sigma 3$ est horizontal et orienté NE-SW (Figure 4). D'après l'analyse microtectonique et la détermination des états de contraintes que nous avons effectué dans tout le Moyen Atlas, cette extension NE-SW est d'âge Miocène supérieur (Hinaje, 2004). Nous pensons qu'à cette époque existait un bassin continental constitué d'un ensemble de grabens NW-SE, sur la transversale Azrou-Timahdite. Ce bassin a été le siège d'une sédimentation 
fluviatile et probablement lacustre, attestée par la présence d'éléments liasiques arrondis dont la taille peut dépasser $1 \mathrm{~m}$. Ce bassin, à sédimentation continentale, est parallèle au bassin de Sefrou-Tahla - Skoura-Tazarine de même direction, mais à sédimentation marine. Ces deux zones effondrées, se sont formées sous un régime extensif NE-SW d'âge miocène supérieur. Au cours de cette phase, apparaissent quelques volcans de type maar (peut être à cause de la présence de plans d'eau lacustres) dont la plupart sont masqués par les coulées volcaniques postérieurs.

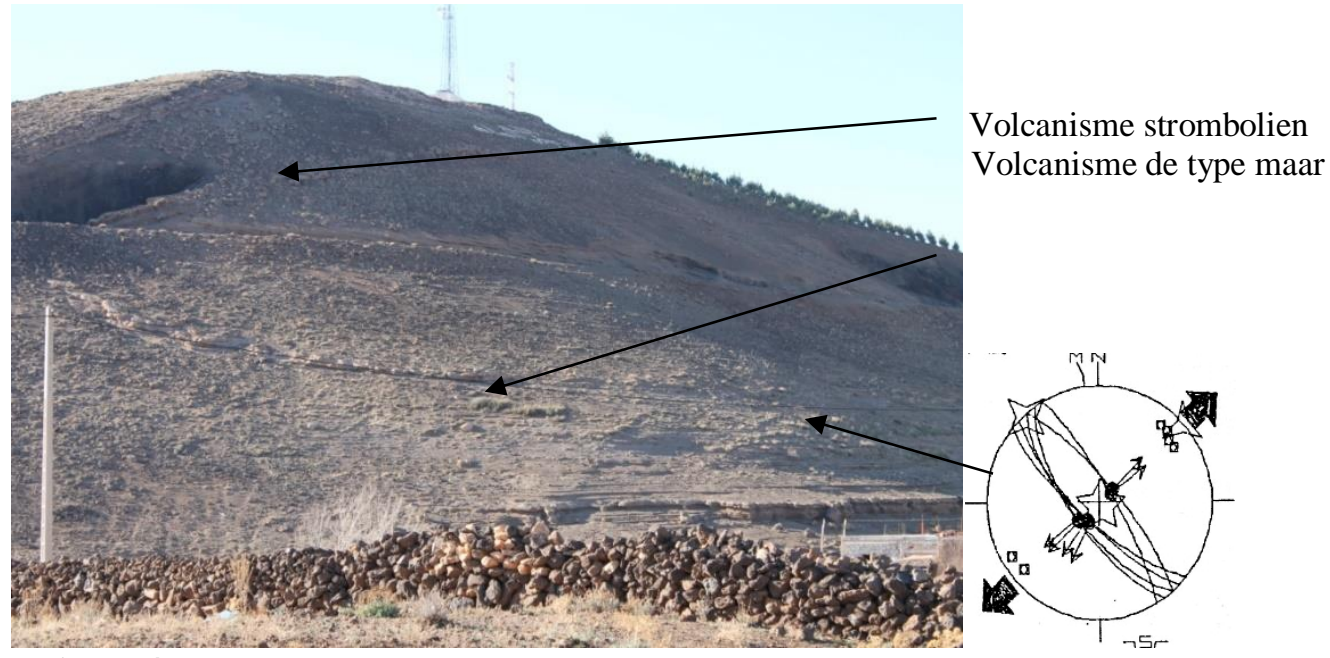

Figure 4 : Volcan de Timahdite de type maar à la base et strombolien au sommet; et stéréogramme des microfailles normales NW-SE synvolcaniques aux dépôts du maar.

Dans le volcan de type maar de Michlifène (voir Figure 1), les dépôts volcano-détritiques sont affectés par une fracturation N60 à N90, qui est localement scellée. L'ensemble est masqué par des coulées basaltiques provenant des volcans avoisinants de forme conique. Les produits volcaniques dans ce maar sont aussi affectés par des microfailles normales subméridiennes, postérieurement aux fractures N60 à N90. Ainsi on peut attribuer au maar Michlifène un âge probable pliocène moyen-supérieur, par analogie avec les dépôts lacustres de Sefrou et de Saïs, attribués au Pliocène moyen-supérieur et affectés par la même phase tectonique synsédimentaire.

\section{Les volcans du Causse d'Anoceur-Zelghi-Tafrawt}

Dans cette zone, les cratères volcaniques sont de petite taille ne dépassant pas une dizaine à une centaine de mètres. Ils correspondent aux volcans les plus septentrionaux du causse moyen atlasique. Cette zone située à la limite NW de l'accident nord moyen atlasique, est affectée par un réseau de failles de directions NE-SW, NW-SE, N-S et ENE-WSW. Parmi les lignes structurales transversales; l'accident orienté N110 à N140 qui se prolonge 
depuis la boutonnière de Kandar jusqu'à Amane Ilila, que nous avons nommé A.K.A.I, recoupe l'A.N.M.A (voir Figure 1). La zone d'intersection de ces deux lignes structurales montre une zone d'ouverture en transtension où siège un volcanisme strombolien récent représenté par le volcan de Jbel Zalghi (Ouaoussenfacht). Le même mécanisme tectonique a provoqué la formation du volcan de type maar de Tafraout-Aït Bouziane, sur la zone en transtension dans le relai de deux décrochements dextres N120 à N140, subparallèles à l'accident de Kandar-Aman Ilila (Figure 5).

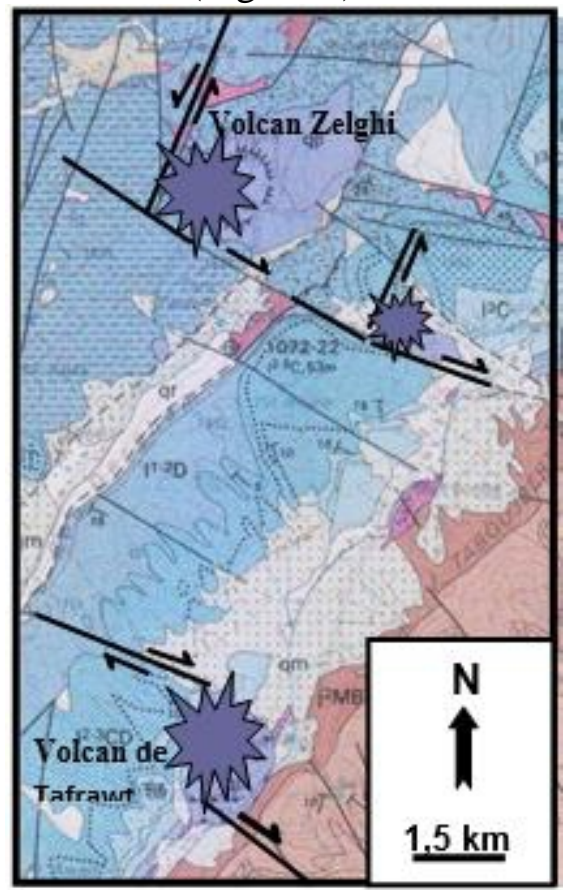

Figure 5 : Position des volcans de Zelghi et de Tafrawt par rapport aux failles longitudinales NE-SW et transversales NW-SE à WNW-ESE (d'après la carte géologique de Sefrou 1/100 000, modifiée).

Les failles les plus récentes à Azghar sont orientées N160 à N10 avec un jeu normal. Ce jeu d'âge quaternaire moyen-récent (Hinaje et al., 2002), est responsable de l'effondrement du fossé de Bou Hda - Boutferoun de direction subméridienne. Dans ce graben, il y a quatre cratères volcaniques qui correspondent probablement à la dernière activité volcanique du Moyen Atlas. Elle est liée à un épisode extensif tel que l'axe $\sigma 1$ est vertical et l'axe $\sigma 3$ est horizontal et orienté ENE-WSW (Figure 6). La lave issue de ces cônes volcaniques, possède encore un champ magnétique, car elle déplace l'aiguille de la boussole d'un angle de $70^{\circ}$. Ces laves basaltiques sont probablement les plus récentes du Moyen Atlas, et méritent donc d'être datées. Elles sont liées au jeu normal des failles subméridiennes qui affectent les carbonates du Lias inférieur et moyen, lors de l'épisode extensif ENE-WSW d'âge quaternaire 
moyen-récent. La présence de failles transverses N140 à jeu décrochant dextre, semble jouer un rôle important dans cette activité volcanique. Le cratère volcanique de Bou Hda est lui-même affecté par des microfailles normales de direction subméridiennes, compatibles avec un deuxième épisode extensif ENE-WSW. Le premier épisode tectonique est anté à synvolcanique, et le deuxième est post-volcanique. Actuellement, la zone est sismiquement active. Une enquête auprès des habitants de la région, nous a révélé qu'un à deux séismes frappent la zone annuellement. Egalement, il faut signaler l'absence de volcans de type maar, probablement du fait de l'inexistence de plans d'eau (lacs) avant et au moment de l'activité volcanique au Quaternaire moyen-récent.

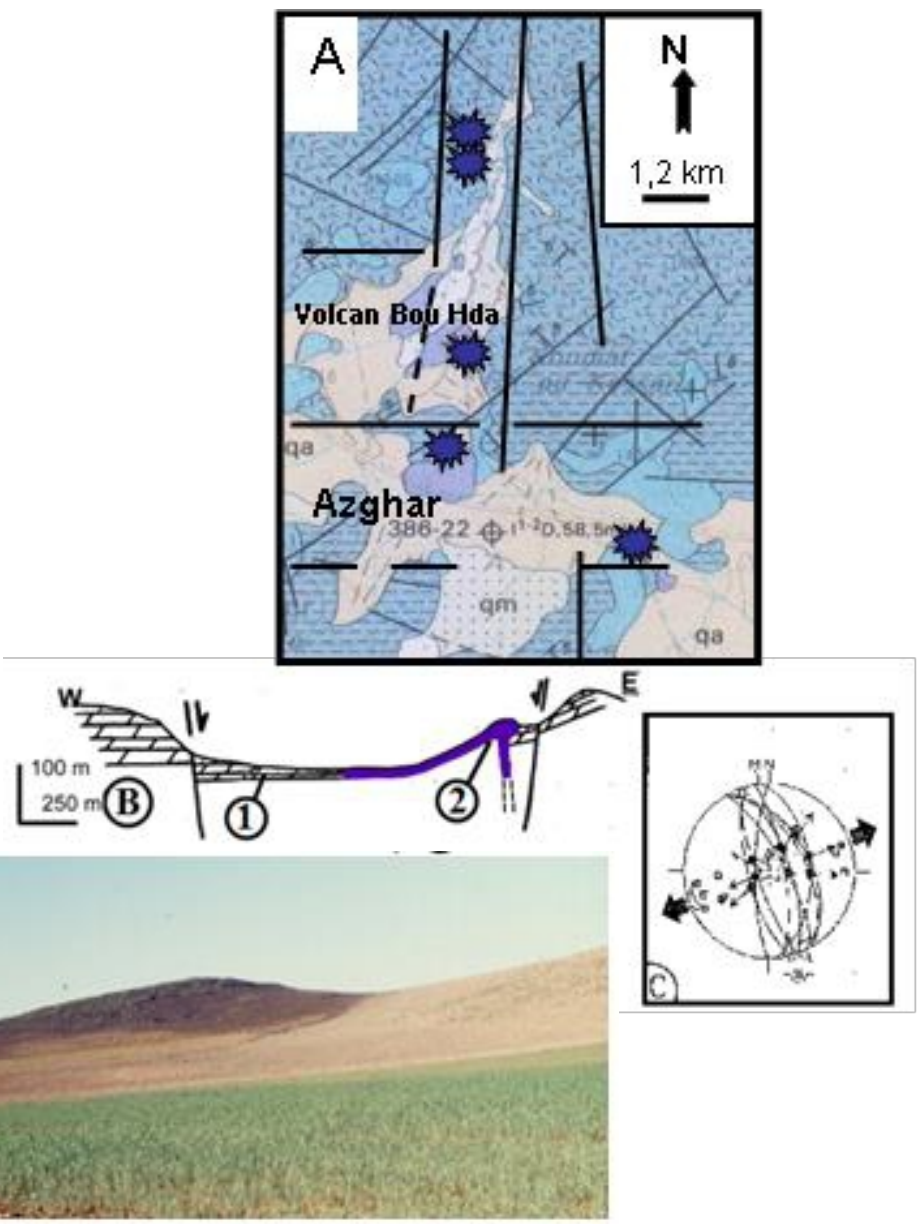

Figure 6: A- Carte des principales failles et des cratères volcaniques dans la zone d'Azghar (d'après la carte géologique de Sefrou 1/100 000, modifiée). B- Coupe structural à travers le volcan de Bou Hda. 1- dolomie du Lias inférieur ; 2- lave et cône volcaniques. C- Analyse microtectonique de la déformation cassante et détermination des axes de contraintes lors de l'épisode tectonique extensif N80 d'âge quaternaire moyen - récent à Azghar. D- Vue panoramique du volcan Bou Hda à Azghar (causse d'Amekla). 
Les deux cratères volcaniques situés vers le sud et le SE de Bou Hda, sont liés aux failles normales ENE-WSW d'âge pliocène moyen-supérieur. Donc ils sont plus anciens que ceux du graben de Bou Hda. D’après la chronologie des phases tectoniques atlasiques (Hinaje, 2004), nous leurs attribuons un âge pliocène moyen-supérieur. Leurs datations absolues ont

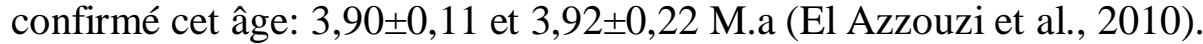

Parmi les volcans du Moyen Atlas méridional, on peut citer celui d'Aguelmam Sidi Ali, près de la zone de passage de l'accident sud moyen atlasique. Celui-ci est divisé en deux cratères de type strombolien, liés à un nœud tectonique où se joignent des failles N130, N50 et N170 (Figure 7). La dépression d'Aguelmam Sidi Ali a été ébauchée au Pliocène moyen-supérieur, puis amplifiée au Quaternaire ancien-moyen, par le jeu normal des failles conjuguées de direction N30 à N50, situées vers le Nord et vers le Sud de ce bassin continental (Hinaje et Aït Brahim ; 2002). L'activité volcanique doit être de même âge (Pliocène moyen-supérieur). Elle est datée à 2,27 $\pm 0,13 \mathrm{M}$.a (El Azzouzi et al., 2010). Elle peut être continue jusqu'au Quaternaire moyenrécent. Au cours de cette dernière période, l'activité tectonique est matérialisée par des failles normales subméridiennes et par un rejeu décrochant senestre des failles NE-SW. La subsidence de la zone d'Aguelmam Sidi Ali a été amplifiée une deuxième fois par le jeu normal des failles subméridiennes au Quaternaire moyen-récent, dont quelques-unes affectent même les basaltes.

Vers le sud-est de la zone d'Aguelmam Sidi Ali, se situent les deux volcans d'Oum Larays et Lougunina, sur un linéament orienté N130. Le volcan Oum Larays a été daté à 0,6 M. a. par la méthode K/Ar sur basaltes (Harmand et Cantagrel, 1984), et à 1,56 — 0,08 M.a (El Azzouzi et al., 2010). Il est situé au niveau de la zone de passage de l'accident d'Aït Oufella (A.S.M.A), dans un relais en transtension avec ouverture, où se joignent une faille normale N40 et un décrochement senestre N70 (Figure 7). Ces jeux sont compatibles avec un état de contrainte d'âge quaternaire ancien-moyen, tels que l'axe $\sigma 1$ est horizontal et orienté N30 et l'axe $\sigma 3$ est aussi horizontal et orienté N120 (avec permutation $\sigma 1-\sigma 2$ ). Au niveau du volcan Lougnina, nous avons remarqué au moins deux activités volcaniques. La première est représentée par des éléments basaltiques contenus dans un conglomérat à ciment calcaire. Sur celui-ci, repose une deuxième lave basaltique plus récente. Une datation absolue a donné un âge de 2,28 — 0,09 M.a (El Azzouzi et al., 2010) correspondant à notre avis, à la première activité volcanique.

Les laves basaltiques très fluides de ces volcans méridionaux, ont suivi les vallées et les dépressions, comme celles du fossé de Guigou qui correspond à un graben effondré au Quaternaire ancien-moyen. Celui-ci a été colmaté par les laves volcaniques provenant des volcans situés au SW à Aguelmam Sidi Ali. Les laves basaltiques dans ce graben sont liées à plusieurs activités 
volcaniques dont la plus récente est intercalée dans des dépôts fluviatiles de basses terrasses holocènes (Amrani et Hinaje, 2014).
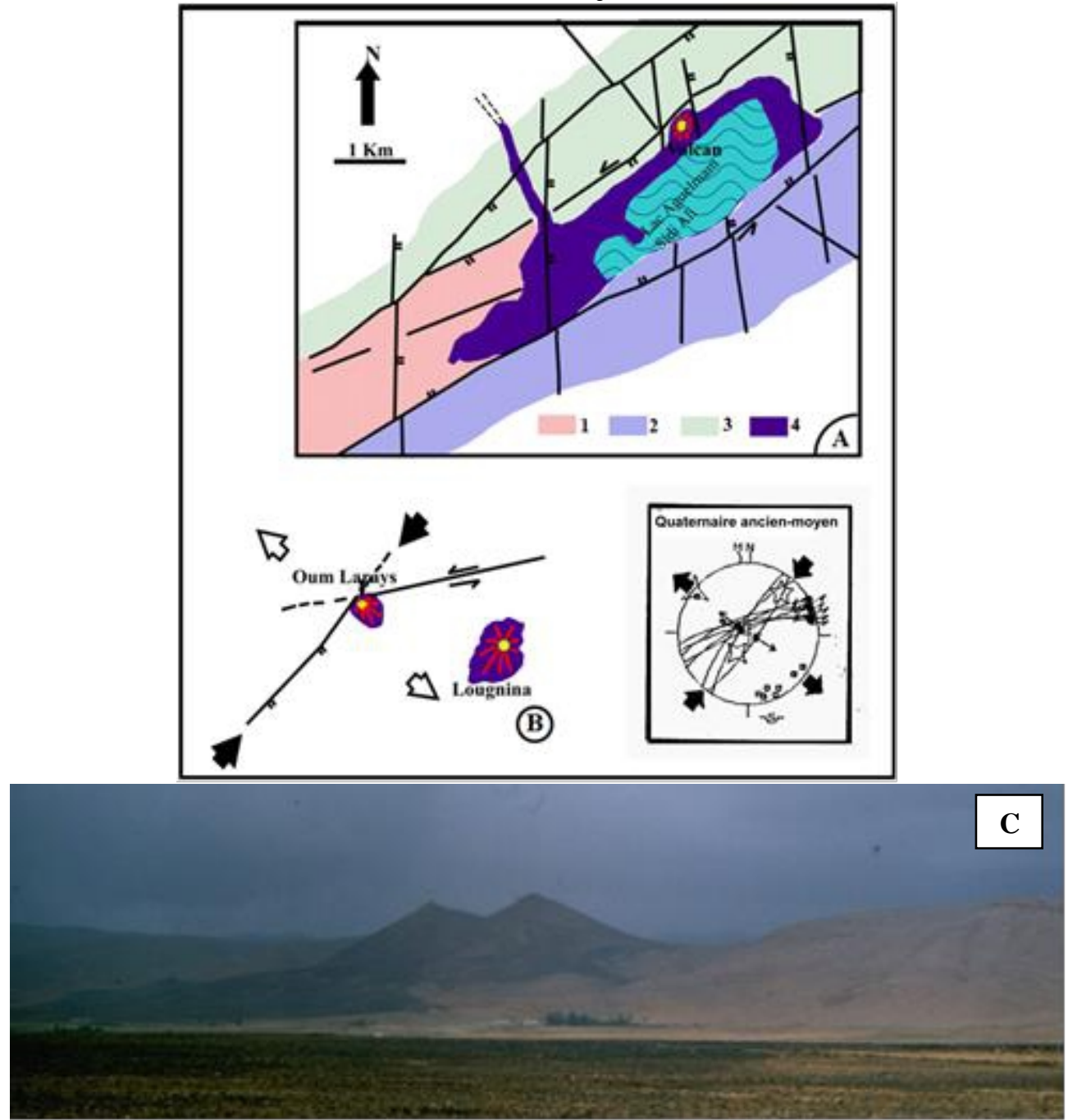

Figure 7: A- Schéma structural de la zone d'Aguelmam Sidi Ali, montrant la situation des laves et du cône volcaniques quaternaires. 1- Trias supérieur-Lias inférieur ; 2- Lias inféreur et moyen ; 3- Bajocien ; 4- laves basaltiques. B- Analyse de la déformation cassante et détermination des axes de contraintes lors de la phase tectonique syn-éruptive d'âge quaternaire ancien-moyen, et modèle tectonique de mise en place du volcan Oum Larays au niveau de la zone de passage de l'accident sud moyen atlasique. C- Vue panoramique du volcan Oum Larays situé sur l'intersection de deux failles; N70 senestre et N30 normale.

\section{Conclusion}

L'analyse microtectonique des failles et leur relation avec l'activité volcanique dans le Moyen Atlas, montre que la répartition spatiale des édifices volcaniques (cônes et maars) est contrôlée par une tectonique cassante polyphasée : 
- $\quad$ la première phase tectonique est responsable de l'effondrement de la zone située entre Timahdit et Azrou, orientée NW-SE, lors d'une extension NE-SW d'âge miocène supérieur. Cet effondrement est attesté par la présence de dépôts détritiques dont les éléments sont de taille centimétrique à métrique et arrondis, emballés dans les dépôts volcano-détritiques de type maar. Ceci témoigne de l'antériorité de l'effondrement et du remplissage de cette zone par rapport à la première activité volcanique. Nous attribuant à cette phase un âge miocène supérieur par analogie aux résultats que nous avons obtenu dans le bassin de Skoura-Tazouta-Sefrou-Tahla. Ce paléochamp de contraintes est probablement contemporain d'une première activité volcanique dont les édifices sont alignés N120 à N140. Cette phase tectonique est représentée par un épisode compressif NW-SE au Pliocène inférieur (permutations des axes $\sigma 1-\sigma 2$ et $\sigma 2-\sigma 3)$;

la deuxième phase correspond à une extension NNW-SSE d'âge pliocène moyen - supérieur, caractérisée par des failles normales N60 à N80. Ces failles affectent les dépôts volcano-sédimentaires de quelques maars comme celui de Michlifène;

la troisième phase dont le champ de contraintes est caractérisé par un axe $\sigma 1$ orienté $\mathrm{N} 30$ et un axe $\sigma 3$ orienté $\mathrm{N} 120$, est responsable de l'effondrement du fossé de l'Oued Guigou à remplissage fluvio-lacustre et basaltique. Au cours de cette phase tectonique les accidents N70 présentent un jeu décrochant senestre, et les accidents N20 à N40 présentent un jeu normal. Les zones de relais en transtension entre ces accidents sont le siège d'activités volcaniques comme le cas du volcan Oum Larays situé sur l'accident sud moyen atlasique. Cette phase tectonique est responsable de la mise en place d'édifices volcaniques alignés selon la direction N30 à N40 ; correspondant à des fentes de tension ou à des «pull-apart » en transtension. Nous attribuons un âge quaternaire ancien à moyen à cet événement, par comparaison à d'autres zones du Moyen Atlas où les dépôts sont datés;

la quatrième phase tectonique d'âge quaternaire moyen - récent dont la contrainte $\sigma 1$ est orientée NNW-SSE et la contrainte $\sigma 3$ est orientée ENEWSW, responsable de la mise en place des derniers édifices volcaniques dont l'alignement général est orienté N170 en moyenne. Ceux-ci correspondent à des méga-fentes de tension, des ouvertures en «pull-apart» et des grabens subméridiens dans un système tectonique en extension ou en transtension. A notre avis les volcans les plus récents sont situés vers le NE (région d'Azghar et causse d'Anoceur) où nous avons dénombré plus de 5 petits cônes volcaniques d'échelle métrique, le long d'un réseau de failles normales subméridiennes.

En raison des relations évidentes qui existent entre le réseau hydrographique actuel, les dernières laves volcaniques et les grabens subméridiens ; les basaltes du Moyen Atlas ont été attribués, à tort, à cette 
dernière phase tectonique. A notre avis, l'activité volcanique dans la chaîne atlasique marocaine est continue depuis l'Eocène (volcan de Zebzate), le Vallésien (volcan de Oued Zra) et jusqu'au Quaternaire moyen-récent (volcans d'Azghar); avec des alignements différents en relation avec une tectonique polyphasée.

\section{References:}

1. Angelier, J. (1975). Sur l'analyse de mesures recueillies dans les sites de failles : l'utilité d'une confrontation entre les méthodes dynamiques et cinématiques. C.R. Acad. Sci., Paris, 181 (D), p.1805-1808.

2. Angelier, J. et Mechler, P. (1977). Sur une méthode graphique de recherche des contraintes principales également utilisable en tectonique et en séismologie : la méthode des dièdres droits. Bull. Soc. Géol. Fr., (7), t, XIX, n 6, pp. 1309-1318.

3. Angelier, J. (1984). Tectonic analysis of fault slip data sets. J. Geophys. Res., 89, B7, p. 5834-5848.

4. Angelier, J. (1989). From orientation to magnitudes in paleostress detrminations using fault slip data. J. Struct. Geol., vol. 11, n 1/2, pp. 37-50.

5. Angelier, J. (1990). Inversion of field data in tectonics to obtain the regional stress - III. A new rapid direct inversion method by analytical means. Geophys. J. Int., 103, p. 363-376.

6. Amrani, S. \& Hinaje, S. (2014). Relation entre la fracturation et la circulation des eaux souterraines dans la zone subsidente Timahdit Almis Guigou (Moyen Atlas, Maroc). Meeting International : La Valorisation des Ressources en Eau Dans les Zones Arides Et Semi Arides. FST Beni Mellal.

7. Bellon, H. (1981). Chronologie radiométrique $\mathrm{K}-\mathrm{Ar}$ des manifestations magmatiques autour de la Méditerranée occidentale entre 33 et $1 \mathrm{Ma}$, in: Wezel F.C. (éd.), sedimentary basins of mediterranean margins, Tecnoprint, Bologne, 341-360.

8. Charrière, A. (1990). Héritage hercynien et évolution géodynamique alpine d'une chaîne intracontinentale : le Moyen Atlas au SE de Fès (Maroc). Thèse Doct. Etat, Toulouse, 589p.

9. Charroud, M. (2002). Evolution géodynamique des Hauts Plateaux (Maroc) et de ses bordures du Mésozoïque au Cénozoïque. Thèse Doc.Etat,Fès, 314p.

10. Chorowicz, J., Emran, A., \& Alem, E. M. (2001). Tectonique et venues volcaniques en contexte de collision, exemple du massif néogène du Siroua (Atlas Marocain): effets combinés d'une transformante et de la suture panafricaine. Canadian Journal of Earth Sciences, 38(3), 411425. 
11. Chotin, P. \& Aït Brahim, L. (1988). Transpression et magmatisme au Néogène-Quaternaire dans le Maroc oriental. C. R. Acad. Sci. Paris, t. 306, Série II, pp. 1479-1485.

12. Choubert, G., Diouri, M. \& Fauire-Muret, A. (1965). Mesures géochronologiques récentes par la méthode A40/K40 au Maroc. Notes Serv. Géol. Maroc 24, 1863, pp.53-62.

13. Duggen, S., Hoernle, K. A., Hauff, F., Kluegel, A., Bouabdellah, M., \& Thirlwall, M. F. (2009). Flow of Canary mantle plume material through a subcontinental lithospheric corridor beneath Africa to the Mediterranean. Geology, 37(3), 283-286.

14. El Azzouzi, M. H., Griffiths, B., Bellon, H., Maury, R. C., Pique, A., Fourcade, S., Cotten, J. et Hernandez, J. (1999). Evolution des sources du volcanisme marocain au cours du Néogène. C. R. Acad. Sci. Paris, 329, pp. 95-102.

15. El Azzouzi, M. H., Maury, R. C., Bellon, H., Youbi, N., Cotten, J., \& Kharbouch, F. (2010). Petrology and K-Ar chronology of the Neogene-Quaternary Middle Atlas basaltic province, Morocco. Bulletin de la société géologique de France, 181(3), 243-257.

16. Frizon de Lamotte, D., Leturmy, P., Missenard, Y., Khomsi, S., Ruiz, G., Saddiqi, O., Guillocheau, F. et Michard, A. (2009). Mesozoic and Cenozoic vertical movements in the Atlas system (Algeria, Morocco, Tunisia): an overview. Tectonophysics, 2009, vol. 475, no 1, p. 9-28.

17. Harmand, C. \& Cantagrel, J. M. (1984). Le volcanisme alcalin tertiaire et quaternaire du Moyen-Atlas (Maroc) : chronologie K/Ar et cadre géodynamique. J. Afric. Earth. Sc., vol. 2, n 1, pp. 51-55.

18. Harmand, C. \& Moukadiri, A. (1986). Synchronisme entre tectonique compressive et volcanisme alcalin: exemple de la province quaternaire du Moyen-Atlas (Maroc). Bull. Soc. Géol. France, II, 4, pp. 595-603.

19. Hernandez, J. \& Bellon, H. (1985). Chronologie K-Ar du volcanisme miocène du Rif oriental (Maroc): implications tectoniques et magmatologiques. Rev. Géol. dyn. Géogr. Phys., vol. XXVI, fas. 2, p. 85-94.

20. Hinaje, S. \& Aït Brahim, L. (2001). Paléocontraintes et contexte géodynamique de mise en place du volcanisme alcalin du Moyen Atlas. Deuxième Colloque 3 M. A. (Fac. Sci. Semlalia, Marrakech).

21. Hinaje, S., Aït Brahim, L. \& Gourari, L. (2002). Rôle de la tectonique cassante polyphasée (du Miocène supérieur à l'actuel) dans la genèse du tracé hydrographique de la vallée de l'Oued Aggay et l'effondrement du bassin de Sefrou (bordure NW du Moyen-Atlas, Maroc). Africa Geoscience Review, Vol. 9, № 2, pp. 171-181. 
22. Hinaje, S. \& Aït Brahim, L. (2002). Les bassins lacustres du Moyen Atlas (Maroc) : un exemple d'activité tectonique polyphasée associée à des structures d'effondrement. Commun. Inst. Geol. e Mineiro, 2002, t. 89 ; pp. $183-184$.

23. Hinaje, S. (2004). Tectonique cassante et paléochamps de contraintes dans le Moyen Atlas et le Haut Atlas central (Midelt-Errachidia) depuis le Trias jusqu'à l'Actuel. Thèse d'Etat Es- Sciences, Rabat, 393 p.

24. Hinaje, S., Charroud, M., Aït Brahim, L., Sabaoui, L., Amrani, S. \& El Arabi, H. (2015). Evénements tectono-sédimentaires, paléocontraintes crétacés-cénozoïques et structuration diachrone du Moyen Atlas et du Haut Atlas central marocains. ScienceLib. Editions Mersenne : 2015 Volume 7, $\mathrm{N}^{\circ}$ 150409, ISSN 2111-4706, 22 p.

25. Jaeger, J. J., Michaux, J. \& David, B. (1973). Biochronologie du Miocène moyen et supérieur continental du Maghreb. C. R. Ac. Sci. Paris, t. 277, p. 2477-2480.

26. Kerchaoui, S. (1994). Pétrologie, géochimie et âges des roches magmatiques. Leur place dans l'évolution structurale du massif des Béni Bou Ifrour et du Rif oriental, Maroc. Thèse phd. Univ. Laval, Québec, 396 p.

27. Martin, J. (1981). Le Moyen Atlas central, étude géomorphologique. Notes et Mém. Serv. Géol. Maroc, n² 258, 445 p.

28. Michard, A. (1976). Elément de géologie marocaine. Notes et mém. serv. Géol. Maroc, 252, 408 p.

29. Moukadiri, A. (1983). Les enclaves ultrabasiques associées aux basaltes alcalins dans le district volcanique d'Azrou-Timahdit (Moyen-Atlas, Maroc). Thèse $3^{\circ}$ cycle, Clermont-Ferrand, $150 \mathrm{p}$.

30. Pique, A. (1994). Géologie du Maroc, les domaines régionaux et leur évolution structurale, pumag, $284 \mathrm{p}$.

31. Pique, A., Aït Brahim, L., El Azzouzi, M'H., Maurey, R., Bellon, H., Semroud, B. \& Laville, E. (1998). Le Poinçon maghrébin: contraintes structurales et géochimiques. Comptes Rendus de l'Académie des Sciences, Paris, 326, p. 575-581.

32. Ramdani, M. et Tadili, B. (1980). Sismicité détaillée du Moyen Atlas et sondages sismiques profonds dans le Maroc central. Thèse 3ème cycle. Univ. Grenoble I, $150 \mathrm{p}$.

33. Termier, H., Termier, G. \& Jouravsky, G. (1948). Une roche volcanique à gros grain de la famille des ijolites: la Talzastite. Notes Serv. géol. Maroc, 1, 71, 81-120.

34. Torbi, A. (1997). Déformations cassantes et paléochamps de contrainte enregistrés dans les terrains méso-cénozoïques du Maroc nordoriental. Thèse Doct. Etat, Oujda, 279 p. 